\title{
QUASI-PERIODIC AND PERIODIC SOLUTIONS FOR VECTOR NONLINEAR SCHRÖDINGER EQUATIONS
}

\author{
J. C. EILBECK, V. Z. ENOLSKII, AND N. A. KOSTOV
}

\begin{abstract}
We consider quasi-periodic and periodic (cnoidal) wave solutions of a set of $n$-component vector nonlinear Schrödinger equations (VNLSE). In a biased photorefractive crystal with a drift mechanism of nonlinear response and Kerr-type nonlinearity, $n$ component nonlinear Schrödinger equations can be used to model self-trapped mutually incoherent wave packets. These equations also model pulse-pulse interactions in wavelength-divisionmultiplexed channels of optical fibre transmission systems. Quasiperiodic wave solutions for the VNLSE in terms of $n$-dimensional Kleinian functions are presented. Periodic solutions in terms of Hermite polynomials and generalized Hermite polynomials for $n$ component nonlinear Schrödinger equations are found.
\end{abstract}

\section{INTRODUCTION}

We consider the system of coupled nonlinear Schrödinger equations

$$
i \frac{\partial}{\partial t} Q_{j}+s \frac{\partial^{2}}{\partial x^{2}} Q_{j}+\sigma\left(\sum_{k=1}^{n}\left|Q_{k}\right|^{2}\right) Q_{j}=0, \quad j=1, \ldots, n,
$$

where $s= \pm 1, \sigma= \pm 1$. These equations are important for a number of physical applications. For example, for photorefractive media with a drift mechanism of nonlinear response, a good approximation describing the propagation of $n$ self-trapped mutually incoherent wave packets is the set of equations for a Kerr-type nonlinearity [29]

$$
i \frac{\partial}{\partial z^{\prime}} \tilde{Q}_{j}+\frac{1}{2} \frac{\partial^{2}}{\partial x^{\prime 2}} \tilde{Q}_{j}+\alpha \delta \eta \tilde{Q}_{j}=0, \quad j=1, \ldots, n,
$$

where $\tilde{Q}_{j}$ denotes the $j$ th component of the beam, $\alpha$ is a coefficient representing the strength of nonlinearity, $z^{\prime}$ and $x^{\prime}$ are the coordinate

The research described in this publication was supported in part by grants from the Bulgarian Civil Research Development Foundation, INTAS grant no. 96-770 (JCE and VZE), the Royal Society (JCE and VZE) and the EPSRC (JCE, VZE, and NAK). 
along the direction of propagation and transverse coordinate respectively. The change in refractive index profile $\eta$ created by all the incoherent components in the light beam is defined by

$$
\delta \eta=\sum_{k=1}^{n}\left|\tilde{Q}_{k}\right|^{2} .
$$

Inserting (1.3) in (1.2) and renormalising the variables as $\tilde{Q}_{j}=Q_{j} / \sqrt{2 \alpha}$, $z^{\prime}=2 t, x^{\prime}=x$ we obtain the vector nonlinear Schrödinger equation (1.1). Stability, localization, and soliton asymptotics of multicomponent photorefractive cnoidal waves are discussed in [35]. New solutions in explicit form are presented here for the case $n=3$. The pulse-pulse collision between wavelength-division-multiplexed channels of optical fibre transmission systems are described with equations of VNLSE type (1.1) $[33,24]$.

Quasi-periodic solutions in terms of $N$-phase theta functions for the Manakov system [30], which is the system (1.1) with $n=2$, are derived in [1], while a series of special solutions are given in [3, 36, 37, 38]. The authors have already discussed quasi-periodic and periodic solutions associated with Lamé and Treibich-Verdier potentials for the Manakov system in the framework of a special ansatz [12]. General quasi-periodic solutions in terms of $N$-phase theta functions of the VNLSE are derived in $[10,28]$ and for matrix nonlinear Schrödinger equations in [46]. We also mention the method of constructing elliptic finite-gap solutions of the stationary KdV and AKNS hierarchy, based on a theorem due to Picard, proposed in [19, 21, 20], as well the method developed by Smirnov in a series of publications: the review paper [40] and [41, 42].

In the present paper, we investigate (1.1) by introducing a special ansatz to analyse hyperelliptic and elliptic solutions of the VNLSE.

The paper is organised as follows. In the Section 2 we construct the Lax representation of the finite dimensional system obtained after substitution of the ansatz and develop a hyperelliptic curve, which are associated with the system. In Section 3 we present the integration of the system in terms of Kleinian hyperelliptic functions, which give quasi-periodic solutions. Recently this realization of Abelian functions was discussed in $[7,8,15,9]$. In Section 4 we show how these quasiperiod solutions reduce in special cases to give periodic solutions of (1.1) in terms of elliptic functions. We explain also in Section 4 the reduction of Kleinian hyperelliptic functions to Hermite and generalized Hermite polynomials.

We seek solution of (1.1) in the following form (e.g. Porubov \& Parker [37]) 


$$
Q_{j}=q_{j}(\zeta) \mathrm{e}^{i \Theta_{j}}, \quad j=1, \ldots n
$$

where $\zeta=x-c t, \Theta_{j}=\Theta_{j}(\zeta, t)$, with $q_{j}, \Theta_{j}$ real. Substituting (1.4) into (1.1) and separating real and imaginary parts by supposing that the functions $\Theta_{j}, j=1, \ldots n$ behave as

$$
\Theta_{j}=\frac{1}{2} s c x+\left(a_{j}-\frac{1}{4} s c^{2}\right) t-s C_{j} \int_{0}^{\zeta} \frac{\mathrm{d} \zeta^{\prime}}{q_{j}\left(\zeta^{\prime}\right)^{2}}+\Theta_{j 0}
$$

we obtain the system $(\sigma=s= \pm 1)[47,26,43,27]$

$$
\frac{d^{2}}{d z^{2}} q_{j}+\left(\sum_{k=1}^{n} \frac{\sigma}{s} q_{k}^{2}-\frac{a_{j}}{s}\right) q_{j}-\frac{C_{j}^{2}}{q_{j}^{3}}=0, \quad k, j=1, \ldots n
$$

where $C_{j}, j=1, \ldots n$ are free parameters and $\Theta_{j 0}$ are constants. These equations describe the integrable case of motion of a particle in a quartic potential perturbed with inverse squared potential, which is separable in ellipsoidal coordinates [47].

\section{LAX REPRESENTATION}

The system (1.5), with $s=1, \sigma=1$ is a completely integrable Hamiltonian system with the Hamiltonian

$$
H=\frac{1}{2} \sum_{i=1}^{n} p_{i}^{2}+\frac{1}{4}\left(\sum_{i=1}^{n} q_{i}^{2}\right)^{2}-\frac{1}{2} \sum_{i=1}^{n} a_{i} q_{i}^{2}+\frac{1}{2} \sum_{i=1}^{n} \frac{C_{i}^{2}}{q_{i}^{2}}
$$

where the variables $\left(q_{i}, p_{i}\right), i=1, \ldots n, p_{i}(\zeta)=\mathrm{d} q_{i}(\zeta) / \mathrm{d} \zeta$, are the canonically conjugated variables with respect to the standard Poisson bracket, $\{\cdot ; \cdot\}$.

This system has the Lax representation [27]

$$
\begin{aligned}
\frac{d L(\lambda)}{d \zeta} & =[M(\lambda), L(\lambda)], \\
L(\lambda) & =\left(\begin{array}{cc}
V(\lambda) & U(\lambda) \\
W(\lambda) & -V(\lambda)
\end{array}\right), \quad M=\left(\begin{array}{cc}
0 & 1 \\
Q(\lambda) & 0
\end{array}\right),
\end{aligned}
$$


which is equivalent to (1.5), where $U(\lambda), W(\lambda), Q(\lambda)$ have the form

$$
\begin{aligned}
& U(\zeta, \lambda)=-a(\lambda)\left(1+\frac{1}{2} \sum_{i=1}^{n} \frac{q_{i}^{2}}{\left(\lambda-a_{i}\right)}\right), V(\zeta, \lambda)=-\frac{1}{2} \frac{d U(\zeta, \lambda)}{d \zeta} \\
& W(\zeta, \lambda)=a(\lambda)\left(-\lambda+\frac{1}{2} \sum_{i=1}^{n} q_{i}^{2}+\frac{1}{2} \sum_{i=1}^{n} \frac{1}{\lambda-a_{i}}\left(p_{i}^{2}+\frac{C_{i}^{2}}{q_{i}^{2}}\right)\right) \\
& Q(\zeta, \lambda)=\lambda-\sum_{i=1}^{n} q_{i}^{2} .
\end{aligned}
$$

The Lax representation yields the hyperelliptic curve $K=(\nu, \lambda)$

$$
\operatorname{det}\left(L(\lambda)-\frac{1}{2} \nu \mathbf{1}_{2}\right)=0,
$$

where $\mathbf{1}_{2}$ is the $2 \times 2$ unit matrix. The moduli of the curve (2.4) generate the integrals of motion $H, F^{(i)}=H_{i}+I_{i}, i=1, \ldots, n$,

$$
\nu^{2}=V^{2}(\zeta, \lambda)+U(\zeta, \lambda) W(\zeta, \lambda) .
$$

The curve (2.5) can be written in canonical form as

$$
\nu^{2}=4 \prod_{j=0}^{2 n}\left(\lambda-\lambda_{j}\right)
$$

where $\lambda_{j} \neq \lambda_{k}$ are branching points.

From (2.5) and explicit expressions for $U(\zeta, \lambda), V(\zeta, \lambda), W(\zeta, \lambda)$ we obtain

$$
\nu^{2}=a(\lambda)^{2}\left(\lambda-\sum_{i=1}^{n} \frac{H_{i}}{\lambda-a_{i}}-\frac{1}{4} \sum_{i=1}^{n} \frac{J_{i}^{2}}{\left(\lambda-a_{i}\right)^{2}}+\sum_{i=1}^{n} \frac{I_{i}}{\lambda-a_{i}}\right),
$$

where

$$
\begin{aligned}
& I_{i}=\frac{1}{4} \sum_{k \neq i} \frac{1}{a_{i}-a_{k}}\left(\left(q_{i} p_{k}-q_{k} p_{i}\right)^{2}-\frac{C_{i}^{2} q_{k}}{q_{i}^{2}}-\frac{C_{k}^{2} q_{i}}{q_{k}^{2}}\right), \\
& H_{i}=\frac{1}{2} p_{i}^{2}-\frac{1}{2} a_{i} q_{i}^{2}+\frac{1}{4} q_{i}^{2}\left(\sum_{k=1}^{n} q_{k}^{2}\right)+\frac{1}{2} \frac{C_{i}^{2}}{q_{i}^{2}}, \\
& J_{i}=2 C_{i}, a(\lambda)=\prod_{i=1}^{n}\left(\lambda-a_{i}\right),
\end{aligned}
$$

and $\sum_{i=1}^{n} H_{i}$ is the Hamiltonian. The parameters $C_{i}$ are linked with the coordinates of the points $\left(a_{i}, \nu\left(a_{i}\right)\right)$ by the formula

$$
C_{i}^{2}=-\frac{\nu\left(a_{i}\right)^{2}}{\prod_{k \neq i}\left(a_{i}-a_{k}\right)},
$$


where $i=1, \ldots n$. Let us define new coordinates $\mu_{i}, i=1, \ldots n$ as zeros of the entry $U(\lambda)$ in the Lax operator. Then

$$
q_{i}^{2}=2 \frac{\prod_{j=1}^{n}\left(a_{i}-\mu_{j}\right)}{\prod_{k \neq i}^{n}\left(a_{i}-a_{k}\right)},
$$

where $i=1, \ldots n$. The definition of $\mu_{i}, i=1, \ldots n$ in combination with the Lax representation leads to the equations

$$
\nu_{i}=V\left(\mu_{i}\right)=-\frac{1}{2} \frac{d}{d \zeta} U\left(\mu_{i}\right), \quad i=1, \ldots, n,
$$

which can be transformed to equations of the form ${ }^{1}$

$$
u_{i}=\sum_{k=1}^{n} \int_{\lambda_{k}}^{\mu_{k}} \mathrm{~d} u_{i}
$$

where $\mathrm{d} u_{i}, i=1, \ldots, n$ denote independent canonical holomorphic differentials

$$
\mathrm{d} u_{i}=\lambda^{i-1} \frac{\mathrm{d} \lambda}{\nu},
$$

and $u_{k}=d_{k}, u_{n}=2 \zeta+b$ with the constants $d_{k}, b, k=1, \ldots, n-1$ defined by the initial conditions. The integration of the problem then reduces to the solution of the Jacobi inversion problem associated with the curve, which consist of the expression of the symmetric functions of $\left(\mu_{i}, \nu_{i}, i=1, \ldots, n\right)$ as function of $n$ complex variables $\left(u_{i}, i=\right.$ $1, \ldots, n)$.

\section{ExaCt SOLUTIONS IN TERMS OF KLEINIAN HYPERELLIPTIC FUNCTIONS}

In this section we give the trajectories of the system under consideration in terms of Kleinian hyperelliptic functions (see, e.g. [4, 8]), associated with the algebraic curve (2.6) of genus $n$ can be also written in the form

$$
\nu^{2}=4 \prod_{j=0}^{2 n}\left(\lambda-\lambda_{j}\right)=4 \lambda^{2 n+1}+\sum_{j=0}^{2 n} \alpha_{j} \lambda^{j} .
$$

At all real branching points $\lambda_{j} \neq \lambda_{k}$ the closed intervals $\left[\lambda_{2 i-1}, \lambda_{2 i}\right], i=$ $1, \ldots n$ will be referred further as lacunae $[48,31]$. Let us equip the curve with a homology basis $\left(\mathfrak{a}_{i} ; \mathfrak{b}_{i}, i=1, \ldots, n\right) \in H_{1}(K, \mathbb{Z})$ and fix

\footnotetext{
${ }^{1}$ In what follows we shall denote the integral bounds by the second coordinate of the curve $K=K(\nu, \lambda)(2.7)$.
} 
the basis in the space of holomorphic differentials. The canonical meromorphic differentials of the second kind $\mathrm{d} \boldsymbol{r}^{T}=\left(\mathrm{d} r_{i}, i=1, \ldots, n\right)$, associated with (2.12), have the form

$$
\mathrm{d} r_{j}=\sum_{k=j}^{2 n+1-j}(k+1-j) \alpha_{k+1+j} \frac{\lambda^{k} d \lambda}{4 \nu}, j=1, \ldots, n .
$$

The $n \times n$ matrices of their periods are

$$
\begin{aligned}
2 \omega & =\left(\oint_{\mathfrak{a}_{k}} \mathrm{~d} u_{l}\right)_{k, l=1, \ldots, n}, \quad 2 \omega^{\prime}=\left(\oint_{\mathfrak{b}_{k}} \mathrm{~d} u_{l}\right)_{k, l=1, \ldots, n}, \\
2 \eta & =\left(-\oint_{\mathfrak{a}_{k}} \mathrm{~d} r_{l}\right)_{k, l=1, \ldots, n}, \quad 2 \eta^{\prime}=\left(-\oint_{\mathfrak{b}_{k}} \mathrm{~d} r_{l}\right)_{k, l=1, \ldots, n} .
\end{aligned}
$$

Define the Abel map $K^{g} \rightarrow \mathbb{C}^{g} /\left(2 \omega \oplus 2 \omega^{\prime}\right)=\operatorname{Jac}(K)$ of symmetrized product of $\mathrm{K}$ to the Jacobi variety whose coordinates $\boldsymbol{u}^{T}=\left(u_{1}, \ldots, u_{g}\right)$ are given by the formula (2.11).

The fundamental $\sigma$ function in this case is a natural generalization of the Weierstrass elliptic $\sigma$ function and is defined as follows

$$
\begin{aligned}
\sigma(\boldsymbol{u})= & \frac{\sqrt{\pi^{n}}}{\sqrt{\operatorname{det}(2 \omega)}} \frac{\epsilon}{\sqrt[4]{\prod_{0 \leq i<j \leq 2 n}\left(\lambda_{i}-\lambda_{j}\right)}} \\
& \times \exp \left\{\boldsymbol{u}^{T} \eta(2 \omega)^{-1} \boldsymbol{u}\right\} \theta[\varepsilon]\left((2 \omega)^{-1} \boldsymbol{u} \mid \omega^{\prime} \omega^{-1}\right),
\end{aligned}
$$

where $\epsilon^{8}=1, \tau=\omega^{\prime} \omega^{-1}$ and $\theta[\varepsilon](\boldsymbol{v} \mid \tau)$ is the $\theta$-function,

$$
\theta[\varepsilon](\boldsymbol{v} \mid \tau)=\sum_{\boldsymbol{m} \in \mathbb{Z}^{n}} \exp i \pi\left\{(\boldsymbol{m}+\boldsymbol{\varepsilon})^{T} \tau(\boldsymbol{m}+\boldsymbol{\varepsilon})+2\left(\boldsymbol{v}+\boldsymbol{\varepsilon}^{\prime}\right)^{T}(\boldsymbol{m}+\boldsymbol{\varepsilon})\right\},
$$

with the half integer characteristic

$$
[\varepsilon]=\left[\begin{array}{ccc}
\varepsilon_{1} & \cdots & \varepsilon_{n} \\
\varepsilon_{1}^{\prime} & \cdots & \varepsilon_{n}^{\prime}
\end{array}\right], \quad \forall \varepsilon_{i}, \varepsilon_{j}=\frac{1}{2} \text { or } 0 .
$$

The characteristic $[\varepsilon]$ is choosen in such the way, that it is the characteristic of the point $\boldsymbol{e}$ at which the $\theta$-function $\theta[0](\boldsymbol{e})$ vanishes to the order $\left[\frac{n+1}{2}\right]$. According to the vanishing Riemann theorem and Clifford theorem the Jacobi variety $\operatorname{Jac}(K)$ of the hyperelliptic curve of genus $n$ always has such the point(see e.g. [17])

The $2 n \times 2 n$ period matrix, $\left(\begin{array}{cc}\omega & \omega^{\prime} \\ \eta & \eta^{\prime}\end{array}\right)$ satisfies the generalized Legendre relation

$$
\left(\begin{array}{cc}
\omega & \omega^{\prime} \\
\eta & \eta^{\prime}
\end{array}\right)\left(\begin{array}{cc}
0 & -1_{g} \\
1_{g} & 0
\end{array}\right)\left(\begin{array}{cc}
\omega & \omega^{\prime} \\
\eta & \eta^{\prime}
\end{array}\right)^{T}\left(\begin{array}{cc}
0 & -1_{g} \\
1_{g} & 0
\end{array}\right)=-\frac{i \pi}{2} .
$$


This relation (3.3) allows us to define a multidimensional $\sigma$-function, which has the following properties

- The $\sigma$-function possesses the following periodicity property: if $\boldsymbol{E}\left(\boldsymbol{m}, \boldsymbol{m}^{\prime}\right)=\eta \boldsymbol{m}+\eta^{\prime} \boldsymbol{m}^{\prime}, \quad$ and $\quad \boldsymbol{\Omega}\left(\boldsymbol{m}, \boldsymbol{m}^{\prime}\right)=\omega \boldsymbol{m}+\omega^{\prime} \boldsymbol{m}^{\prime}$, where $\boldsymbol{m}, \boldsymbol{m}^{\prime} \in \mathbb{Z}^{n}$, then

$$
\begin{aligned}
& \sigma[\varepsilon]\left(\boldsymbol{z}+2 \boldsymbol{\Omega}\left(\boldsymbol{m}, \boldsymbol{m}^{\prime}\right), \omega, \omega^{\prime}\right)=\exp \left\{2 \boldsymbol{E}^{T}\left(\boldsymbol{m}, \boldsymbol{m}^{\prime}\right)\left(\boldsymbol{z}+\boldsymbol{\Omega}\left(\boldsymbol{m}, \boldsymbol{m}^{\prime}\right)\right)\right\} \\
& \quad \times \exp \left\{-\pi i \boldsymbol{m}^{T} \boldsymbol{m}^{\prime}-2 \pi i \boldsymbol{\varepsilon}^{T} \boldsymbol{m}^{\prime}\right\} \sigma[\varepsilon]\left(\boldsymbol{z}, \omega, \omega^{\prime}\right) .
\end{aligned}
$$

- As a modular function, the Kleinian $\sigma$-function is invariant under the transformation of the symplectic group, which represents an important characteristic feature.

We introduce the following notation. Kleinian $\zeta$ and $\wp$-functions are defined as logarithmic derivatives of the fundamental $\sigma$-function

$$
\begin{gathered}
\zeta_{i}(\boldsymbol{u})=\frac{\partial \ln \sigma(\boldsymbol{u})}{\partial u_{i}}, \quad i=1, \ldots, n ; \\
\wp_{i j}(\boldsymbol{u})=-\frac{\partial^{2} \ln \sigma(\boldsymbol{u})}{\partial u_{i} \partial u_{j}}, \wp_{i j k}(\boldsymbol{u})=-\frac{\partial^{3} \ln \sigma(\boldsymbol{u})}{\partial u_{i} \partial u_{i} \partial u_{k}}, \ldots, \\
i, j, k, \ldots=1, \ldots, n .
\end{gathered}
$$

The functions $\zeta_{i}(\boldsymbol{u})$ and $\wp_{i j}(\boldsymbol{u})$ have the following periodicity properties

$$
\begin{aligned}
\zeta_{i}\left(\boldsymbol{u}+2 \Omega\left(\boldsymbol{m}, \boldsymbol{m}^{\prime}\right)\right) & =\zeta_{i}(\boldsymbol{u})+2 E_{i}\left(\boldsymbol{m}, \boldsymbol{m}^{\prime}\right), \quad i=1, \ldots, n, \\
\wp_{i j}\left(\boldsymbol{u}+2 \Omega\left(\boldsymbol{m}, \boldsymbol{m}^{\prime}\right)\right) & =\wp_{i j}(\boldsymbol{u}), \quad i, j=1, \ldots, n,
\end{aligned}
$$

where $E_{i}\left(\boldsymbol{m}, \boldsymbol{m}^{\prime}\right)$ is the $i$-th component of the vector $E\left(\boldsymbol{m}, \boldsymbol{m}^{\prime}\right)=$ $\eta \boldsymbol{m}+\eta^{\prime} \boldsymbol{m}^{\prime}$ and $\boldsymbol{\Omega}\left(\boldsymbol{m}, \boldsymbol{m}^{\prime}\right)=\omega \boldsymbol{m}+\omega^{\prime} \boldsymbol{m}^{\prime}$.

Alternatively the $\sigma$ function can be defined by its expansion near $\boldsymbol{u}=0$. In particular, for small genera we have

$\sigma(\boldsymbol{u})=u_{1}+o\left(\boldsymbol{u}^{3}\right)$ for $n=1$ and 2 ,

$\sigma(\boldsymbol{u})=u_{1} u_{3}-u_{2}^{2}+o\left(\boldsymbol{u}^{4}\right)$ for $n=3$ and 4 ,

$\sigma(\boldsymbol{u})=-u_{3}^{3}+2 u_{2} u_{3} u_{4}-u_{1} u_{4}^{2}-u_{2}^{2} u_{5}+u_{1} u_{3} u_{5}+o\left(\boldsymbol{u}^{5}\right)$ for $n=5$ and 6 .

and further terms can be computed with the help of a bilinear differential equation [5].

The principal result of the theory is the formula of Klein, which reads in the case of genus $n$ as follows

$$
\sum_{k, l=1}^{n} \wp_{k l}\left(\int_{\infty}^{\mu} \mathrm{d} \mathbf{u}-\sum_{m=1}^{n} \int_{a_{m}}^{\mu_{m}} \mathrm{~d} \mathbf{u}\right) \mu^{k-1} \mu_{i}^{l-1}
$$




$$
=\frac{F\left(\mu, \mu_{i}\right)+2 \nu \nu_{i}}{4\left(\mu-\mu_{i}\right)^{2}}, \quad i=1, \ldots n
$$

where

$$
F\left(\mu_{1}, \mu_{2}\right)=\sum_{r=0}^{n} \mu_{1}^{r} \mu_{2}^{r}\left[2 \alpha_{2 r}+\alpha_{2 r+1}\left(\mu_{1}+\mu_{2}\right)\right] .
$$

By expanding these equalities in the neighbourhood of infinity we obtain the complete set of the relations for the hyperelliptic functions.

The first group of relations represents the solution of the Jacobi inversion problem in the form

$$
\lambda^{n}-\wp_{n n}(\boldsymbol{u}) \lambda^{n-1}-\wp_{n, n-1}(\boldsymbol{u}) \lambda^{n-2}-\ldots-\wp_{n 1}(\boldsymbol{u})=0 .
$$

Let us introduce a polynomial $\mathcal{F}(\boldsymbol{u}, \lambda)$ in $\lambda$ of degree $n$

$$
\mathcal{F}=\lambda^{n}-\wp_{n, n}(\boldsymbol{u}) \lambda^{n-1}-\wp_{n, n-1}(\boldsymbol{u}) \lambda^{n-2}-\ldots-\wp_{n, 1}(\boldsymbol{u}) .
$$

which are solutions of the following nonlinear equation

$$
2\left(\frac{d^{2}}{d u_{n}^{2}} \mathcal{F}\right) \mathcal{F}-\left(\frac{d}{d u_{n}} \mathcal{F}\right)^{2}-4(\lambda+U) \mathcal{F}^{2}+\nu^{2}=0
$$

where $\mathrm{U}=2 \wp_{n n}+\frac{1}{4} \alpha_{2 n}$. It is possible to show that the following equations are valid

$$
\wp_{n n n i}=\left(6 \wp_{n n}+\alpha_{2 n}\right) \wp_{n i}+6 \wp_{n, i-1}-2 \wp_{n-1, i}+\frac{1}{2} \delta_{n i} \alpha_{2 n-1},
$$

for $i=1, \ldots n$. These equations can be identified with the KdV hierarchy with "time variables" $\left(t_{1}, t_{2}, \ldots\right)=\left(u_{n}, u_{n-1}, \ldots\right)=(x, t, \ldots)$,

$$
\mathcal{X}_{k+1}[\mathrm{U}]=\mathcal{R} \mathcal{X}_{k}[\mathrm{U}]
$$

where $\mathcal{R}=\partial_{x}^{2}-\mathbf{U}+c-\frac{1}{2} \mathrm{U}_{x} \partial^{-1}$, and $c=\alpha_{2 n} / 12$ is the Lenard recursion operator. The first two equations from the hierarchy are

$$
\mathrm{U}_{t_{1}}=\mathrm{U}_{x}, \quad \mathrm{U}_{t_{2}}=\frac{1}{2}\left(\mathrm{U}_{x x x}-6 \mathrm{U}_{x} \mathrm{U}\right)
$$

the second equation is the $\mathrm{KdV}$ equation, which is obtained from (3.9) with $i=n$ as the result of differentiation by $x=u_{n}$ and setting $\mathrm{U}=$ $2 \wp_{n n}+\frac{1}{6} \alpha_{2 n}$. The next group of equations are obtained from $(3.7,3.8)$ 
and for simplicity we present results only for genus $n=3$

$$
\begin{aligned}
\wp_{333}^{2}= & 4 \wp_{33}^{3}+\alpha_{6} \wp_{33}^{2}+4 \wp_{23} \wp_{33}+\alpha_{5} \wp_{33}+4 \wp_{22}-4 \wp_{13}+\alpha_{4}, \\
\wp_{233}^{2}= & 4 \wp_{23}^{2} \wp_{33}+\alpha_{6} \wp_{23}^{2}-4 \wp_{22} \wp_{23}+8 \wp_{13} \wp_{23}+4 \wp_{11}+\alpha_{2}, \\
\wp_{133}^{2}= & 4 \wp_{13}^{2} \wp_{33}+\alpha_{6} \wp_{13}^{2}-4 \wp_{12} \wp_{13}+\alpha_{0}, \\
\wp_{233} \wp_{333}= & 4 \wp_{33}^{2} \wp_{23}+\alpha_{6} \wp_{23} \wp_{33}-2 \wp_{22} \wp_{33}, \\
& +4 \wp_{13} \wp_{33}-2 \wp_{23}^{2}+\frac{1}{2} \alpha_{5} \wp_{23}+2 \wp_{12}+\frac{1}{2} \alpha_{3}, \\
\wp_{133} \wp_{233}= & 4 \wp_{13} \wp_{23} \wp_{33}+\alpha_{6} \wp_{13} \wp_{23}-2 \wp_{12} \wp_{23}-2 \wp_{13} \wp_{22}+4 \wp_{13}^{2}+\frac{1}{2} \alpha_{1}, \\
\wp_{133} \wp_{333}= & 4 \wp_{13} \wp_{33}^{2}+\alpha_{6} \wp_{13} \wp_{33}-2 \wp_{12} \wp_{33}+2 \wp_{13} \wp_{23}+\frac{1}{2} \alpha_{5} \wp_{13}-2 \wp_{11} .
\end{aligned}
$$

Let us introduce finally the Baker-Akhiezer function, which in the framework of the formalism developed is expressible in terms of the Kleinian $\sigma$-function as follows

$$
\Psi(\lambda, \boldsymbol{u})=\frac{\sigma\left(\int_{\infty}^{\lambda} \mathrm{d} \boldsymbol{u}-\mathbf{u}\right)}{\sigma(\boldsymbol{u})} \exp \left\{\int_{\infty}^{\lambda} \mathrm{d} \mathbf{r}^{T} \boldsymbol{u}\right\},
$$

where $\lambda$ is arbitrary and $\boldsymbol{u}$ is the Abel image of an arbitrary point $\left(\nu_{1}, \mu_{1}\right) \times \ldots \times\left(\nu_{n}, \mu_{n}\right)$. It is straightforward to show by direct calculation, using the relations for three and four-index Kleinian $\wp$-functions, that $\Psi(\lambda, \boldsymbol{u})$ satisfy the Schrödinger equation

$$
\left(\frac{d^{2}}{d u_{n}{ }^{2}}-2 \wp_{n n}(\boldsymbol{u})\right) \Psi(\lambda, \boldsymbol{u})=\left(\lambda+\frac{1}{4} \alpha_{2 n}\right) \Psi(\lambda, \boldsymbol{u})
$$

for all $(\nu, \mu)$.

Now we are in a position to write the solution of the of the system in terms of Kleinian $\sigma$-functions and identify the constants in terms of the moduli of the curve. Using $(3.7,2.9)$, the solutions of (1.5) have the following form in terms of Kleinian functions $\wp_{n m}(\boldsymbol{u})$, $\left.\wp_{n(m-1)}(\boldsymbol{u}), \ldots, \wp_{n 1}(\boldsymbol{u})\right), m=n, \ldots 1$

$$
q_{i}^{2}=2 \frac{a_{i}^{n}-\wp_{n, n}(\boldsymbol{u}) a_{i}^{n-1}-\wp_{n, n-1}(\boldsymbol{u}) a_{i}^{n-2}-\ldots-\wp_{n, 1}(\boldsymbol{u})}{\prod_{k \neq i}\left(a_{i}-a_{k}\right)},
$$

where the vector $\boldsymbol{u}^{T}=\left(d_{j}, 2 \zeta+b\right), j=1, \ldots, n-1$. Finally, the solutions of the VNLSE (1.1) reads in this case

$$
Q_{i}(x, t)=\sqrt{2 \frac{\mathcal{F}\left(\boldsymbol{u}, a_{i}\right)}{\prod_{k \neq i}^{n}\left(a_{i}-a_{k}\right)}} \exp \left(\Theta_{i}\right),
$$


where

$$
\Theta_{j}=\left\{\frac{1}{2} i c x+i\left(a_{j}-\frac{1}{4} c^{2}\right) t-\frac{1}{2} \nu\left(a_{j}\right) \int_{0}^{\zeta} \frac{\mathrm{d} \zeta^{\prime}}{\mathcal{F}\left(\boldsymbol{u}, a_{j}\right)}\right\}
$$

$j=1, \ldots, n$, and we have made use of (3.14) and (1.4).

\section{Elliptic PERIODiC SOlutions}

In this section we develop a method (see also $[26,16,14,11]$ ) which allows us to construct periodic solutions of (1.5) in a straightforward way, based on the application of the spectral theory for the Schrödinger equation with elliptic potentials $[2,31]$. We start with the formula $(2.9)$ and with the equation for the Baker function $\Psi(\lambda ; \boldsymbol{u})$.

$$
\frac{\mathrm{d}^{2}}{\mathrm{~d} x^{2}} \Psi(\lambda, \boldsymbol{u})-U \Psi(x, \boldsymbol{u})=\left(\lambda+\frac{\alpha_{2 n}}{4}\right) \Psi(\lambda, \boldsymbol{u}),
$$

where we identify the potential

$$
\mathrm{U}=2 \wp_{n n}+\frac{1}{6} \alpha_{2 n}
$$

We assume, without loss of generality, that the associated curve has the property $\alpha_{2 n}=0$. To make this assumption applicable to the initial curve of the system (1.5) derived from the Lax representation, we shift the spectral parameter

$$
\lambda \longrightarrow \lambda+\Delta, \quad \Delta=\frac{2}{2 n+1} \sum_{i=1}^{n} a_{i} .
$$

Suppose, that $\mathrm{U}$ is a three, four gap Lamé or three, four gap TreibichVerdier potential, which means that

$$
\mathrm{U}(x)=2 \sum_{i=1}^{N} \wp\left(x-x_{i}\right)
$$

where $\wp(x)$ is the standard Weierstrass elliptic function with periods $2 \omega, 2 \omega^{\prime}$ and $x_{i}$ takes values from the set $\left\{0, \omega_{1}=\omega, \omega_{2}=\omega+\omega^{\prime}, \omega_{3}=\right.$ $\left.\omega^{\prime}\right\}$ It is known, that the set of such potentials is exhausted by the 
following potentials for genus $n=3,4[44,40,21]$

$$
\begin{aligned}
& \mathrm{U}(x)=12 \wp(x) \\
& \mathrm{U}(x)=12 \wp(x)+2 \wp\left(x+\omega_{i}\right), \quad i=1,2,3 \\
& \mathrm{U}(x)=6 \wp(x)+6 \wp\left(x+\omega_{i}\right)+2 \wp\left(x+\omega_{j}\right), \quad i \neq j=1,2,3 \\
& \mathrm{U}(x)=12 \wp(x)+6 \wp\left(x+\omega_{i}\right), \quad i=1,2,3 \\
& \mathrm{U}(x)=12 \wp(x)+2 \sum_{i=1}^{3} \wp\left(x+\omega_{i}\right) \\
& \mathrm{U}(x)=12 \wp(x)+6 \sum_{i=1}^{2} \wp\left(x+\omega_{i}\right)+2 \wp\left(x+\omega_{3}\right) \\
& \mathrm{U}(x)=20 \wp(x), \\
& \mathrm{U}(x)=12 \wp(x)+6 \sum_{i=1}^{3} \wp\left(x+\omega_{i}\right)
\end{aligned}
$$

The potential (4.4), (4.10) is three, four gap Lamé potential respectively; the potentials $(4.5,4.7,4.8,4.6,4.11,4.9)$ are Treibich-Verdier potentials $[44,39,40]$.

To display the class of periodic solutions of system (1.5) we introduce the generalized Hermite polynomial $\mathcal{F}(x, \lambda)$ by the formula

$$
\mathcal{F}(x, \lambda)=\lambda^{n}-\pi_{n, n}(x) \lambda^{n-1}-\pi_{n, n-1}(x) \lambda^{n-2}-\ldots-\pi_{n, 1}(x) .
$$

In the particular case of the Lamé potential

$$
\mathrm{U}(x)=n(n+1) \wp(x),
$$

the generalized Hermite polynomial coincide with the standard Hermite polynomial [45]

$$
\mathcal{F}(x, \wp)=\sum_{r=0}^{n} c_{r}(\lambda)\left(\wp(x)-e_{2}\right)^{n-r},
$$

where the coefficients $c_{r}$ are solutions of the following recurrence relation

$$
\begin{aligned}
& 4 r\left(n-r+\frac{1}{2}\right)(2 n-r+1) c_{r} \\
& \quad=(n-r+1)\left[12 e_{2}(n-r)(n-r+2)-4 e_{2}\left(n^{2}+n-3\right)-4 \lambda\right] c_{r-1} \\
& \quad-2(n-r+1)(n-r+2)(2 n-2 r+3)\left(e_{1}-e_{2}\right)\left(e_{2}-e_{3}\right) c_{r-2} .
\end{aligned}
$$

This recurrent formula is satisfied if we set for $r>n+2$

$$
c_{n+1}=c_{n+2} \ldots=0, \quad \text { and normalize } c_{0}=1 .
$$

The associated Lamé curve is defined by (2.6), where the $\lambda_{j}$ are solutions of recurrence relations given in [18], cf. [45]. The original approach 
presented in [18] is useful because the Lamé curve appears in factorised form.

The solutions of the system (1.5) are then given as

$$
q_{i}^{2}(\zeta)=2 \frac{\mathcal{F}\left(\zeta, a_{i}-\Delta\right)}{\prod_{k \neq i}^{n}\left(a_{i}-a_{k}\right)}, i=1, \ldots, n .
$$

The final formula for the solutions of the system (1.1) then reads

$$
Q_{i}(x, t)=\sqrt{2 \frac{\mathcal{F}\left(\zeta, a_{i}-\Delta\right)}{\prod_{k \neq i}^{n}\left(a_{i}-a_{k}\right)}} \exp \left(\Theta_{i}\right),
$$

where

$$
\Theta_{j}=\left\{\frac{1}{2} i c x+i\left(a_{j}-\frac{1}{4} c^{2}\right) t-\frac{1}{2} \nu\left(a_{j}-\Delta\right) \int_{0}^{\zeta} \frac{\mathrm{d} \zeta^{\prime}}{\mathcal{F}\left(\zeta^{\prime}, a_{j}-\Delta\right)}\right\},
$$

and $i=1, \ldots, n$ and we have made use of (4.15) and (1.4).

We shall consider below examples of genus three, four curves, which are associated with the three, four gap elliptic potentials (4.4), (4.10) and $(4.5,4.7,4.8,4.6,4.11,4.9)$.

Consider the potential (4.4) and construct the associated curve [23]

$$
\nu^{2}=4 \lambda \prod_{i=1}^{3}\left(\lambda^{2}-6 e_{i} \lambda+45 e_{i}^{2}-15 g_{2}\right) .
$$

The Hermite polynomial $\mathcal{F}(\wp(x), \lambda)$ associated with the Lamé potential (4.4) has the form

$$
\begin{gathered}
\mathcal{F}(\wp(x), \lambda)=\lambda^{3}-6 \wp(x) \lambda^{2}-3 \cdot 5\left(g_{2}-3 \wp^{2}\right) \lambda- \\
\frac{3^{2} \cdot 5^{2}}{4}\left(4 \wp^{3}-g_{2} \wp-g_{3}\right) .
\end{gathered}
$$

Then the finite and real solution of the system (1.5) is given by (4.16) with the Hermite polynomial depending on the argument $x+\omega^{\prime}$ (the shift in $\omega^{\prime}$ provides the holomorphity of the solution). The solution is real under the choice of the arbitrary constants $a_{i}, i=1, \ldots, n$ in such a way, that the constants $a_{i}-\Delta, i=1, \ldots, n$ lie in different lacunae. According to (1.4) the constants $C_{i}$ are then given as

$$
C_{i}^{2}=-\frac{\nu\left(a_{i}-\Delta\right)^{2}}{\prod_{k \neq i}\left(a_{i}-a_{k}\right)},
$$

where $i=1, \ldots, n$ and $\Delta$ is the shift (4.2) and $\nu$ is the coordinate of the curve (4.17). 
The Hermite polynomial $\mathcal{F}(\wp(x), \lambda)$ associated with the Lamé potentials can be written in a different form, useful for applications:

$$
\mathcal{F}(\wp(x), \lambda)=\sum_{k=0}^{n} A_{k}(\lambda) \wp(x)^{n-k} .
$$

For example for the genus $n=4$ Lamé potential (4.10) we have

$$
\begin{aligned}
& A_{0}=11025, \quad A_{1}=-1575 \lambda, \quad A_{2}=135 \lambda^{2}-\frac{6615}{2} g_{2} \\
& A_{3}=-10 \lambda^{3}+\frac{1855}{4} \lambda g_{2}-2450 g_{3} \\
& A_{4}=\lambda^{4}-\frac{113}{2} \lambda^{2} g_{2}+\frac{3969}{16} g_{2}^{2}+\frac{1925}{4} \lambda g_{3} .
\end{aligned}
$$

For the general Lamé potential the associated curve is defined by

$$
\begin{aligned}
\nu^{2}(\lambda) & =-4 \lambda A_{n}^{2}-A_{n} A_{n-1} g_{2}+\left(A_{n-1}^{2}-4 A_{n-2} A_{n}\right) g_{3} \\
& =4 \prod_{j=0}^{2 n}\left(\lambda-\lambda_{j}\right) .
\end{aligned}
$$

For the genus four Lamé potential (4.10) we have

$$
\nu^{2}=\left(\prod_{l=1}^{3}\left(\lambda^{2}+10 e_{l} \lambda-7\left(5 e_{l}^{2}+g_{2}\right)\right)\right)\left(\lambda^{3}-52 \lambda g_{2}+560 g_{3}\right) .
$$

To display the class of periodic solutions of the system (1.5) for genus $n=3$ we express the generalized Hermite polynomial $\mathcal{F}(x, \lambda)$ by the formula

$$
\mathcal{F}(x, \lambda)=\lambda^{3}-\pi_{33}(x) \lambda^{2}-\pi_{23}(x) \lambda-\pi_{13}(x)
$$

with $\pi_{33}(x), \pi_{23}(x)$ and $\pi_{13}(x)$ given as follows

$$
\begin{aligned}
\pi_{33}(x)= & \sum_{j=1}^{N} \wp\left(x-x_{j}\right)+\frac{1}{3} \sum_{j=0}^{6} Z_{j}, \\
\pi_{12}(x)= & -3 \sum_{i<j} \wp\left(x-x_{i}\right) \wp\left(x-x_{j}\right)-\frac{N g_{2}}{8} \\
& \quad-\frac{1}{6} \sum_{i<j} \lambda_{i} \lambda_{j}+\frac{1}{6}\left(\sum_{j=0}^{6} \lambda_{j}^{2}\right) \\
\pi_{13}(x)= & \frac{5}{2} \pi_{33}^{3}-\frac{5}{4} \pi_{33 ; x x} \pi_{33}+\frac{1}{8} \pi_{33} \alpha_{5}-\frac{5}{8} \pi_{33 ; x}^{2}+\frac{1}{16} \pi_{33 ; x x x x}-\frac{1}{8} \alpha_{4},
\end{aligned}
$$

where $x_{i}$ are half-periods, $N$ is the degree of the cover, $Z_{j}, j=0, \ldots 6$ are potential shifts (see for example [16]). Without loss of generality we 
assume that $\alpha_{6}=\sum_{j=0}^{6} \lambda_{j}=0$. For conciseness we denote $\wp=\wp(x)$, $\wp\left(x+\omega_{1}\right)=\wp_{1}$. Then for the Treibich-Verdier potential (4.5) we find

$$
\begin{aligned}
\pi_{33}= & 6 \wp+\wp_{1}-e_{1} \\
\pi_{23}= & -45 \wp^{2}-18 \wp \wp_{1}+18 \wp_{1}+3 \wp_{1} e_{1}+ \\
& +\frac{7}{4} g_{2}+156 e_{1}^{2} \\
\pi_{13}= & 225 \wp^{3}+225 \wp^{2} \wp_{1}-90 \wp \wp_{1} e_{1}-\frac{15}{2} \wp_{x} \wp_{1 ; x}- \\
& -225 \wp^{2} e_{1}+\frac{105}{4} \wp g_{2}+\frac{5}{4} \wp_{1} g_{2}-900 \wp e_{1}^{2}- \\
& -150 \wp_{1} e_{1}^{2}+\frac{6237}{2} e_{2}+155 e_{1}^{3}-\frac{2079}{2} g_{2}-\frac{7}{4} g_{2} e_{1}-\frac{451}{8} g_{3},
\end{aligned}
$$

and the associated Treibich-Verdier curve has the form [40]

$$
\begin{aligned}
\nu^{2}=\quad & 4\left(\lambda-5\left(e_{1}-e_{2}\right)\right)\left(\lambda-5\left(e_{1}-e_{3}\right)\right) \\
& \left(\lambda^{2}+12 \lambda e_{1}-20\left(e_{1}-e_{2}\right)\left(e_{1}-e_{3}\right)\right) \\
& \left(\lambda^{3}+3 e_{1} \lambda^{2}+\left(-141 e_{1}^{2}-4 g_{2}\right)+45 e_{1}\left(e_{1}-4 e_{2}\right)\left(5 e_{1}+4 e_{2}\right) .\right.
\end{aligned}
$$

The full list of generalized Hermite polynomials for other TreibichVerdier potentials will be published elsewhere.

\section{Conclusions}

In this paper we have described a family of elliptic solutions for the vector nonlinear Schrödinger equations using a Lax pair method and the general method of reduction of Abelian functions to elliptic functions. Our approach is systematic in the sense that special solutions (periodic, soliton, etc.) are obtained in a unified way.

In fibre optics applications, periodic and quasi-periodic waves are of interest in optical transmission systems.

\section{REFERENCES}

[1] M. R. Adams, J. Harnad, and J. Hurtubise. Darboux coordinates and Liouville-Arnold integration in loop algebras. Commun. Math. Phys., 155:385-415, 1993.

[2] H. Airault, H. P. McKean, and J. Moser. Rational and elliptic solutions of the KdV equation and a related many-body problem. Comm. Pure and Appl. Math., 30:94-148, 1977.

[3] E. Alfinito, M. Leo, G. Soliani, and L. Solombrino. Symmetry propeties and exact patterns in birefrigent optical fibers. Phys, Rev., 53E(3):3159-3165, 1995.

[4] H. F. Baker. Abels theorem and the allied theory including the theory of theta functions. Cambridge Univ. Press, Cambridge, 1897. 
[5] H. F. Baker. Multiply Periodic Functions. Cambridge Univ. Press, Cambridge, 1907.

[6] E. D. Belokolos and V. Z. Enolskii. Isospectral deformations of elliptic potentials. Russian Math. Surveys, 44(5):155-156, 1989.

[7] V. M. Buchstaber, V. Z. Enolskii, and D. V. Leykin. Kleinian functions, hyperelliptic Jacobians and applications. In S. P. Novikov and I. M. Krichever, editors, Reviews in Mathematics and Mathematical Physics, volume 10:2, pages 1-125, Gordon and Breach: London, 1997.

[8] V. M. Buchstaber, V. Z. Enolskii, and D. V. Leykin. Recursive family of polynomials generated by Sylvester's identity and addition theorem for hyperelliptic Kleinian functions. Funkt. Analiz. Pril, 31(4):19-32, 1997.

[9] V. M. Buchstaber and V. Z. Enolskii and D. V. Leykin. Rational analogues of the abelian functions. Funkt. Analiz. Pril, 33(2):1-15, 1999.

[10] I. V. Cherednik. Differential equations of the Baker-Akhiezer functions of algebraic curves. Funct. Anal. Appl., 12:195-203, 1978.

[11] P. L. Christiansen, J. C. Eilbeck, V. Z. Enolskii, and N. A. Kostov. Quasi periodic solutions of coupled nonlinear Schrödinger equations. Proc. R. Soc. Lond. A, 451:685-700, 1995.

[12] P. L. Christiansen, J. C. Eilbeck, V. Z. Enolskii, and N. A. Kostov. Quasiperiodic and periodic solutions for coupled nonlinear Schrödinger equations of Manakov type. Proc. R. Soc. Lond. A, in press, 2000.

[13] B. Crosignani, A. Cutolo, and P. di Porto. Coupled-mode theory of nonlinear propagation in multimode and single-mode fibres: envelope solitons and selfconfinement. J. Opt. Soc. Am., 72:1136-1141, 1982.

[14] J. C Eilbeck and V. Z. Enolskii. Elliptic Baker-Akhiezer functions and an application to an integrable dynamical system. J. Math. Phys., 35(3):1192$1201,1994$.

[15] J. C Eilbeck, V. Z. Enolskii, and D. V. Leykin. On the Kleinian construction of Abelian functions of canonical algebraic curve. In Proceedings of the Conference SIDE III: Symmetries of Integrable Differences Equations, Saubadia, May 1998, CRM Proceedings and Lecture Notes, pages 121-138, 2000.

[16] V. Z. Enolskii and N. A. Kostov. On the geometry of elliptic solitons. Acta Applicandae Math., 36:57-86, 1994.

[17] H. M. Farkas and I. Kra. Riemann Surfaces, Springer, New York, 1980

[18] F. Gesztesy and R. Weikard. Lamé potentials and the stationary (m)KdV hierarchy. Math. Nachr, 176:73-91, 1995.

[19] F. Gesztesy and R. Weikard. Picard potentials and Hill's equation on a torus. Acta Math., 176:73-107, 1996.

[20] F. Gesztesy and R. Weikard. A characterization of all algebro-geometric solutions of the AKNS hierarchy. Acta Math., 181:63-108, 1998.

[21] F. Gesztesy and R. Weikard. Elliptic algebro-geometric solutions of the KdV and AKNS hierarchies - an analytic approach. Bul.(New Series) AMS, 35:271-317, 1998.

[22] A. Hasegawa and Y. Kodama. Solitons in Optical Communications. Clarendon, Oxford, 1995.

[23] C. Hermite. Oeuvres de Charles Hermite. Vol. III . Gautheir-Villars, Paris, 1912.

[24] Y. Kodama. The Whitham equations for optical communications: mathematical theory of NRZ. solv-int 9709012, 1997. 
[25] Y. Kodama, A. Maruta, and S. Wabnitz. Minimum channel spacing in wavelength-division-multiplexed nonreturn-to-zero optical fiber transmissions. Opt. Lett., 21:1815-1817, 1996.

[26] N. A. Kostov. Quasi-periodical solutions of the integrable dynamical systems related to Hill's equation. Lett. Math. Phys., 17:95-104, 1989.

[27] N. A. Kostov. Korteweg-de Vries hierarchy and related completely integrable systems: I. Algebro-geometrical approach. Preprint INRNE, TH-98/4, 1998, solv-int 9904016.

[28] I. M. Krichever. Spectral theory of "finite-gap" nonstationary Schrödinger operators. Nonstationary Peierls model. Funk. Analiz. Pril., 20:42-54, 1986 (in Russian).

[29] V. Kutuzov, V. M. Petnikova, V. V. Shuvalov, and V. A. Vysloukh. Crossmodulation coupling of incoherent soliton models in photorefractive crystals. Phys. Rev. E, 57:6056-6065, 1998.

[30] S. V. Manakov. On the theory of two-dimensional stationary self-focusing of electromagnetic waves. Zh. Eksp. Teor. Fiz [Sov. Phys. JETP], 65 [38]:505516 [248-253], 1973 [1974].

[31] H. P. McKean and P. van Moerbeke. The spectrum of Hill's operator. Invent. Math., 30:217-274, 1975.

[32] C. R. Menyuk. Nonlinear pulse-propagation in birefringent optical fibers. IEEE J. Quant. Electron., QE-23:174-176, 1987.

[33] L. F. Mollenauer, S. G. Evangelides, and J. P. Gordon. Wavelength division multiplexing with solitons in ultra-long transmission using lumped amplifiers. J. Lightwave Technol., 9:362-367, 1991.

[34] S. P. Novikov. Periodic problem for the Korteweg de Vries equation. Funk. Analiz. Pril., 74:54-66, 1974.

[35] V. M. Petnikova, V. V. Shuvalov, and V. A. Vysloukh. Multicomponent photorefractive cnoidal waves: Stability, localization, and soliton asymptotics. Phys. Rev. E, 60:1009-1018, 1999.

[36] C. Polymilis, K. Hizanidis, and D. J. Frantzeskakis. Phase plane Stäckel potential dynamics of the Manakov system. Phys. Rev., E58:1112-1124, 1998.

[37] A. V. Porubov and D. F. Parker. Some general periodic solutions to coupled nonlinear Schrödinger equation. Wave Motion, 29:97-109, 1999.

[38] V. I. Pulov, I. M. Uzunov, and E. J. Chakarov. Solutions and laws of conservation for coupled nonlinear Schrödinger equations: Lie group analysis. Phys. Rev., E57:3468-3477, 1998.

[39] A. O. Smirnov. Elliptic solutions of the KdV equation. Math. Zametki, 45(6):66-73, 1989.

[40] A. O. Smirnov. Finite-gap elliptic solutions of the KdV equation. Acta Applicandae Math., 36:125-166, 1994.

[41] A. O. Smirnov. 3-elliptic solutions of sine-Gordon equation. Matem. Zametki, 62(3):440, 1997.

[42] A. O. Smirnov. On some set of elliptic potentials of Dirac operator. Math. USSR Sbornik, 188(1):109-128, 1997.

[43] G. Tondo. On the integrability of stationary and restricted flows of the KdV hierarchy. J. Phys. A: Mat. Gen, 28:5097-5115, 1995.

[44] A. Treibich and J. L. Verdier. Revêtements tangentiels et sommes de 4 nombres triangulaires. Comptes Rendus Acad. Sci. Paris, 311:51-54, 1990. 
[45] E. T. Whittaker and G. N. Watson. A Course of Modern Analysis. Cambridge University Press, Cambridge, 1986.

[46] M. A. Wisse, Quasiperiodic solutions for the matrix nonlinear Schrödinger equations. J. Math. Phys., 31:3694-3699, 1992.

[47] S. Wojciechowski. Integrability of one particle in a perturbed central quartic potential. Physica Scripta, 31:433-438, 1985.

[48] V. E. Zakharov, S. V. Manakov, S. P. Novikov, and L. P. Pitaevskii. Soliton theory: inverse scattering method. Nauka, Moscow, 1980.

Department of Mathematics, Heriot-Watt University, Edinburgh, EH14 4AS, UK

E-mail address: J.C.Eilbeck@ma.hw.ac.uk

Department of Mathematics, Heriot-Watt University, Edinburgh, EH14 4AS, UK, And, Theoretical Physics Division, NASU Institute of Magnetism, 36-B Vernadsky str., Kiev-680, 252142, Ukraine

E-mail address: vze@imag.kiev.ua

Institute of Electronics, Bulgarian Academy of Sciences, Blvd.

Tsarigradsko SHOSse 72, Sofia 1784,, Bulgaria

E-mail address: nakostov@ie.bas.bg 